\title{
3 \\ INTERPRETATION OF TRANSMISSION ELECTRON MICROGRAPHS
}


Already published in this series

1. The Operation and Calibration of the Electron Microscope

2. Electron Diffraction in the Electron Microscope

In preparation

4. Typical Electron Microscope Investigations 
Philips Technical Library

Monographs in Practical Electron Microscopy

in Materials Science

\section{3 \\ INTERPRETATION OF \\ TRANSMISSION ELECTRON \\ MICROGRAPHS}

\section{J. W. EDINGTON}

Department of Metallurgy and Materials Science, University of Cambridge, Cambridge, England 
(C) N.V. Philips' Gloeilampenfabrieken, Eindhoven, 1975

Reprint of the original edition 1975

All rights reserved. No part of this publication may be reproduced or transmitted, in any form or by any means, without permission.

This book is sold subject to the standard conditions of the Net Book Agreement.

SBN 333185757

ISBN 978-1-349-02660-9 ISBN 978-1-349-02658-6 (eBook)

DOI 10.1007/978-1-349-02658-6

First published 1975 by

THE MACMILLAN PRESS LTD

London and Basingstoke

Associated companies in New York, Dublin,

Melbourne, Johannesburg and Madras

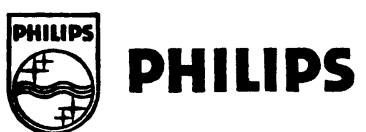

Trademarks of Philips' Gloeilampenfabrieken 


\section{PREFACE}

This is the third of a series of monographs on electron microscopy aimed at users of the equipment. They are written as both texts and sources of reference emphasising the applications of electron microscopy to the characterisation of materials.

In some places the author has referred the reader to material appearing in other monographs of this series. The following titles have already been published:

1. The Operation and Calibration of the Electron Microscope

2. Electron Diffraction in the Electron Microscope and in preparation is:

4. Typical Electron Microscope Investigations

BF Bright field (image)

DF Dark field (image)

CDF Centred dark field (image)

WB Weak beam (image)

DP Diffraction pattern

SADP Selected area diffraction pattern

SRO Short-range order

APB Antiphase domain boundary

$g$ A vector normal to the reflecting plane

$s \quad$ The deviation from the Bragg reflection position

$\xi_{g} \quad$ The extinction distance

$w \quad s \xi_{g}$

b The Burgers vector of an undissociated dislocation

$b_{p}$ The Burgers vector of a partial dislocation

$\boldsymbol{\mu} \quad$ The line vector of a dislocation

FN The upward drawn foil normal (normal to the specimen surface)

B The upward drawn incident beam direction in the specimen, that is opposite to the direction of the electron beam

ANO Anomalous absorption coefficient 


\section{CONTENTS}

\section{INTERPRETATION OF TRANSMISSION ELECTRON MICROGRAPHS}

3.1 IMAGE CONTRAST

\section{DIFFRACTION CONTRAST}

3.2 SUMMARY OF THEORY

3.2.1 The Perfect Crystal 2

$\begin{array}{ll}3.2 .2 & \text { Absorption }\end{array}$

3.2.3 Characteristic Images of Perfect Crystals 3

$\begin{array}{lll}\text { 3.2.3.1 Thickness fringes } & 3\end{array}$

$\begin{array}{lll}\text { 3.2.3.2 } & \text { Bend extinction contours } & 3\end{array}$

$\begin{array}{lll}\text { 3.2.3.3 } & \text { Bend contours } & 5\end{array}$

$\begin{array}{lll}\text { 3.2.3.4 Bend centres } & 5\end{array}$

3.2.4 Crystal Containing a Defect with a Strain Field 5

3.3 RELEVANCE OF DIFFRACTION THEORY TO STUDIES OF CRYSTAL DEFECTS

3.3.1 Experimental Conditions for Quantitative Analysis 9

3.3.2 Recognition of Dynamical and Kinematical Diffracting Conditions in the Electron Microscope

II. QUANTITATIVE ANALYSIS OF CRYSTAL DEFECTS 10

$\begin{array}{llr}3.4 & \text { DISLOCATIONS } & 10\end{array}$

$\begin{array}{lll}3.4 .1 & \text { A Simple Treatment of Dislocation Images } & 10\end{array}$

$\begin{array}{ll}3.4 .2 & 11\end{array}$

$\begin{array}{ll}\text { 3.4.2.1 Elastically isotropic materials } & 11\end{array}$

3.4.2.2 Elastically anisotropic materials $\quad 12$

3.4.3 Determination of the Burgers Vector of Perfect Dislocations 14

$\begin{array}{lll}\text { 3.4.3.1 Elastically isotropic materials } & 14\end{array}$

3.4.3.2 Elastically anisotropic materials $\quad 15$

3.4.4 Images of Partial Dislocations $\left(b_{p}<\right.$ Lattice Translation Vector) 17

$\begin{array}{lll}\text { 3.4.4.1 Fault invisible } & 17\end{array}$

$\begin{array}{llr}\text { 3.4.4.2 } & \text { Fault visible } & 19\end{array}$

3.4.4.3 A partial dislocation between extrinsic and intrinsic faults 20

$\begin{array}{ll}\text { 3.4.5 Determination of the Burgers Vector of Partial Dislocations } & 20\end{array}$

3.4.6 Image Characteristics of Slightly Dissociated Partial Dislocations 22

3.4.7 Overall Procedure for Determining the Burgers Vector of Dislocations 22

$\begin{array}{ll}\text { 3.4.8 Image Characteristics of the Special Case of End-on Dislocations } & 22\end{array}$

3.4.9 Determination of the Sense of the Burgers Vector of End-on Dislocations 23

$\begin{array}{lll}\text { 3.4.10 Image Characteristics of Dislocation Pairs } & 23\end{array}$

$\begin{array}{ll}\text { 3.4.11 Image Characteristics of Dislocation Dipoles } & 23\end{array}$

$\begin{array}{ll}\text { 3.4.12 Faulted Dipoles } & 26\end{array}$

$\begin{array}{lll}3.5 & \text { DISLOCATION LOOPS } & 26\end{array}$

$\begin{array}{lll}3.5 .1 & \text { Large Loops (Diameter } \gtrsim 500 \AA) & 26\end{array}$

$\begin{array}{ll}\text { 3.5.2 Quantitative Information } & 28\end{array}$

3.5.3 Image Characteristics of Medium-sized Loops (Diameter 100-500 §)

$\begin{array}{ll}\text { 3.5.4 Quantitative Information } & 30\end{array}$

3.5.5 Image Characteristics of Small Loops (Diameter $\$ 100 \AA$ )

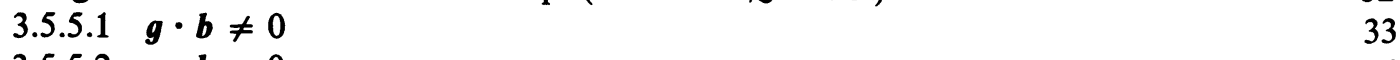

$\begin{array}{lll}3.5 .5 .2 g \cdot b=0 & 36\end{array}$

3.5.6 Quantitative Information $\quad 36$

$\begin{array}{lll}\text { 3.5.6.1 Determination of the Burgers vector } & 36\end{array}$

3.5.6.2 Determination of the vacancy/interstitial sense of the loop 36 
$3.6 \propto$ BOUNDARIES, STACKING FAULTS, PLANAR PRECIPITATES 37

$\begin{array}{ll}\text { 3.6.1 Image Characteristics of Stacking Faults } & 37\end{array}$

3.6.1.1 Single stacking faults $\alpha=0, \pm 2 \pi / 3, \pm 4 \pi / 3, \pm 2 \pi$

3.6.1.2 Overlapping stacking faults 38

3.6.2 Quantitative Information on Stacking Faults $\quad 39$

$\begin{array}{lll}\text { 3.6.2.1 Identification } & 39\end{array}$

3.6.2.2 Determination of the direction of the displacement $R_{\mathrm{n}}$

3.6.2.3 Determination of the extrinsic/intrinsic nature 40

$\begin{array}{ll}\text { 3.6.3 Image Characteristics of Plate Precipitates } & 41\end{array}$

3.6.4 Quantitative Information on Plate Precipitates 41

$3.7 \pi$ BOUNDARIES: ANTIPHASE DOMAIN BOUNDARIES 41

$\begin{array}{llr}3.7 .1 & \text { Image Characteristics } & 43\end{array}$

$\begin{array}{lll}\text { 3.7.1.1 Single boundaries } & 43\end{array}$

3.7.1.2 Overlapping boundaries $\quad 45$

$\begin{array}{ll}\text { 3.7.2 Quantitative Information } & 45\end{array}$

$\begin{array}{lll}3.8 & \delta & \text { BOUNDARIES }\end{array}$

$\begin{array}{ll}\text { 3.8.1 Image Characteristics } & 45\end{array}$

3.8.1.1 Single boundaries $\quad 45$

$\begin{array}{lll}3.8 .1 .2 & \text { Overlapping boundaries } & 47\end{array}$

$\begin{array}{ll}\text { 3.8.2 Quantitative Information } & 49\end{array}$

$\begin{array}{lll}3.9 & \text { MIXED } \alpha-\delta \text { BOUNDARIES } & 49\end{array}$

$\begin{array}{llr}3.9 .1 & \text { Image Characteristics } & 49\end{array}$

$\begin{array}{ll}\text { 3.9.2 Quantitative Information } & 49\end{array}$

3.10 INTERFACES

3.10.1 Image Characteristics of Interfaces Between Like Crystal Structures 49

$\begin{array}{lll}\text { 3.10.1.1 Low-angle boundaries } & 49\end{array}$

3.10.1.2 High-angle boundaries $\quad 52$

3.10.1.3 Twin boundaries $\quad 52$

3.10.2 Quantitative Information on Interfaces Between Like Crystal Structures 55

3.10.2.1 Low-angle boundaries $\quad 55$

3.10.2.2 High-angle boundaries $\quad 55$

$\begin{array}{lll}\text { 3.10.2.3 Twin boundaries } & 55\end{array}$

3.10.3 Image Characteristics of Precipitate-Matrix Interfaces $\quad 56$

$\begin{array}{lll}\text { 3.10.3.1 Coherent interfaces } & 56\end{array}$

3.10.3.2 Partially coherent interfaces $\quad 56$

$\begin{array}{lll}\text { 3.10.3.3 Incoherent interfaces } & 57\end{array}$

3.10.4 Quantitative Information on Precipitate-Matrix Interfaces $\quad 57$

$\begin{array}{lll}\text { 3.10.4.1 Coherent interfaces } & 57\end{array}$

$\begin{array}{lll}\text { 3.10.4.2 } & \text { Partially coherent interfaces } & 57\end{array}$

$\begin{array}{lll}3.10 .4 .3 & \text { Incoherent interfaces } & 59\end{array}$

3.11 STRAIN FIELD IMAGES FROM SMALL MISFITTING PRECIPITATES

$\begin{array}{llr}3.11 .1 & \text { Image Characteristics } & 59\end{array}$

$\begin{array}{llr}3.11 .1 .1 & P_{\mathrm{s}} \lesssim 0.2 & 59\end{array}$

$\begin{array}{lll}3.11 .1 .2 & P_{\mathrm{s}} \gtrsim 0.2 & 60\end{array}$

3.11.2 Quantitative Information $\quad 61$

3.11.2.1 Determination of the sense of the strain field 61

3.11.2.2 Estimation of the magnitude of the misfit $\varepsilon \quad 63$

3.11.3 Image Characteristics of Misfitting Plate Precipitates 64

3.11.4 Quantitative Information on Misfitting Plate Precipitates 65

$\begin{array}{lll}3.12 & \text { CAVITIES } & 65\end{array}$

$\begin{array}{lll}3.12 .1 & \text { Image Characteristics } & 65\end{array}$

$\begin{array}{ll}\text { 3.12.2 Quantitative Information } & 67\end{array}$ 
3.13 GAS BUBBLES $\quad 67$

$\begin{array}{lll}\text { 3.13.1 Image Characteristics } & 67\end{array}$

$\begin{array}{ll}\text { 3.13.2 Quantitative Information } & 67\end{array}$

3.14 VISIBILITY OF DEFECTS IN STRAIN FIELD DIFFRACTION CONTRAST IMAGES 67

3.15 DIFFERENTIATION BETWEEN SIMILAR TYPES OF IMAGES 68

3.16 MANY-BEAM EFFECTS $\quad 68$

3.16.1 Extinction Distance $\quad 68$

$\begin{array}{lll}3.16 .2 & \text { Images } & 68\end{array}$

$\begin{array}{lll}\text { 3.16.2.1 Perfect crystals } & 69\end{array}$

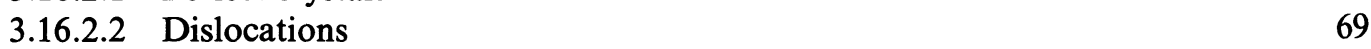

$\begin{array}{lll}\text { 3.16.2.3 } & \text { Misfitting precipitates } & 69\end{array}$

$\begin{array}{lll}\text { 3.16.2.4 Stacking faults } & 69\end{array}$

3.17 WEAK BEAM IMAGES

3.17.1 Image Characteristics $\quad 70$

$\begin{array}{lll}\text { 3.17.1.1 Dislocations } & 70\end{array}$

$\begin{array}{lll}\text { 3.17.1.2 Dislocation loops } & 74\end{array}$

$\begin{array}{lll}3.17 .2 & \text { Applications } & 74\end{array}$

III. PHASE CONTRAST 75

$\begin{array}{lll}3.18 & \text { SUMMARY OF THEORY } & 75\end{array}$

IV. APPLICATIONS OF PHASE CONTRAST TO STUDIES OF CRYSTALS 76

3.19 LATtiCe PLANE RESOlution (TWO- OR THREE-beAm IMAGeS) 76

3.19.1 The Perfect Crystal 76

$\begin{array}{lll}\text { 3.19.2 Crystal Imperfections } & 77\end{array}$

3.20 MULTI-BEAM LATTICE IMAGeS

3.21 MOIRÉ PATTERNS $\quad 80$

$\begin{array}{llr}3.21 .1 & \text { Parallel } & 82\end{array}$

$\begin{array}{lll}3.21 .2 & \text { Rotational } & 82\end{array}$

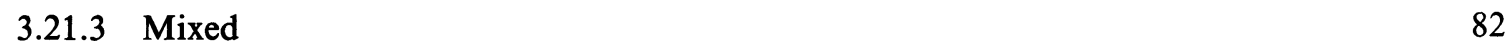

3.21.4 Magnification $\quad 82$

3.21 .5 Summary $\quad 82$

3.21.6 Applications $\quad 84$

3.21.7 Detection $\quad 84$

3.22 DARK FIELD IMAGES OF SHORT-RANGE ORDERED (SRO) OR AMORPHOUS MATERIALS

3.23 MAGNETIC DOMAIN BOUNDARIES (LORENTZ MICROSCOPY)

3.23.1 Types of Domain Boundary

3.23.2 Diffraction Pattern Effects $\quad 86$

3.23.3 Image Characteristics 86

$\begin{array}{lll}\text { 3.23.3.1 } & \text { Fresnel images (out of focus) } & 86\end{array}$

$\begin{array}{lll}\text { 3.23.3.2 } & \text { Foucault images (in focus) } & 88\end{array}$

$\begin{array}{lll}\text { 3.23.3.3 Diffraction contrast images } & 88\end{array}$

$\begin{array}{lll}\text { 3.23.4 Applications } & 88\end{array}$

$\begin{array}{lll}\text { 3.23.4.1 Determination of magnetisation direction } & 89\end{array}$

$\begin{array}{ll}\text { 3.23.4.2 Measurement of domain wall thickness } & 89\end{array}$

3.23.4.3 Determination of magnetisation $\quad 90$

$\begin{array}{ll}\text { 3.23.4.4 Determination of Curie temperature } & 90\end{array}$

3.23.4.5 Determination of anisotropy constant $K \quad 90$

3.23.5 Observations of Magnetic Flux Lines 91

3: Recommended Reading 91

3: References $\quad 92$ 
APPENDIX 3. ELASTIC CONSTANTS 97

Appendix 3: References 101

APPENDIX 8. ATOMIC SCATTERING AMPLITUDES 103

Appendix 8: Reference 103

APPENDIX 10. EXTINCTION DISTANCES IN SOME COMMON MATERIALS 105

Appendix 10: References 105

APPENDIX 11. VALUES OF $\boldsymbol{g} \cdot \boldsymbol{b}$ FOR PERFECT DISLOCATIONS IN f.c.c. 107 AND c.p.h. CRYSTAL STRUCTURES

APPENDIX 12. PREPARATION OF HIGH-RESOLUTION TEST SPECIMENS 109

$\begin{array}{lll}\text { A12.1 SINGLE-CRYSTAL (100) GOLD FILMS } & 109\end{array}$

A12.2 PARTIALLY GRAPHITISED CARBON BLACK 109

A12.3 REPLICAS FOR POINT-TO-POINT RESOLUTION TESTS 109

Appendix 12: References

APPENDIX 13. HOLEY CARBON REPLICAS 112

Appendix 13: References 112 